\title{
Nützt Datenqualität wirklich im CRM? - Wirkungszusammenhänge und Implikationen
}

\author{
Bernd Heinrich
}

Markus Helfert

Zusammenfassung: In den letzten Jahren wird vielfach in Wissenschaft und Praxis unter dem Begriff des Customer Relationship Management (CRM) eine nachhaltig kundenorientierte Ausrichtung von Unternehmen diskutiert. Allerdings scheitern, nach anfänglich großen Erwartungen, zahlreiche CRM-Projekte. Eine überschätzte bzw. schlechte Datenqualität ist dabei sehr häufig als einer der Gründe angeführt, wobei zwar ein Zusammenhang zwischen CRM und Datenqualität unterstellt, jedoch nicht begründet wird. Ziel des Beitrags ist es deshalb, den Wirkungszusammenhang zwischen Kundenbeziehung und Datenqualität modellgestützt zu erklären. Dadurch lässt sich zeigen, dass mit Qualitätsinvestitionen nicht per se der gewünschte Bindungseffekt erzielt wird. Neben dem wissenschaftlichen Nutzen einer derartigen Fundierung soll damit auch eine konzeptionelle Basis für die zukünftige Ableitung praktischer Handlungsempfehlungen gelegt werden.

Schlüsselworte: Datenqualität, Kundenbeziehung, Datenqualitätswirkung, Customer Relationship Management

\section{Einleitung}

Der Aspekt der Datenqualität wird im Kontext des CRM immer wieder ins Feld geführt, wenn es darum geht, den Erfolg - aber auch das Scheitern - entsprechender Projekte zu erklären [z.B. ArLa02; Nels02]. Doch in welchen Fällen und wie wirkt sich eine Verbesserung der Datenqualität auf die Kundenbindung im Sinne einer längeren und intensiveren Beziehung überhaupt aus? Gerade wenn man dem CRM eine so große Bedeutung beimisst, wie es in Forschung und Praxis derzeit getan wird, ist es notwendig derartige Wirkungszusammenhänge zu untersuchen und modellgestützt darzustellen. Deshalb wird zu dieser Problemstellung mit der vorliegenden Arbeit ein grundlagenorientierter Beitrag geleistet, auch um in einem Folgeschritt praktische Handlungsempfehlungen abzuleiten. 
Zu Beginn erfolgt eine Reflektion bisheriger Arbeiten, mittels derer nicht nur die Problemstellung motiviert, sondern gleichermaßen auch der Forschungsbedarf bestimmt wird. Dieser liegt nicht nur in einer für den Beitrag geeigneten Definition von Datenqualität und Kundenbeziehung begründet. Vielmehr soll gezeigt werden, in welchem Bezug die beiden Aspekte stehen und dass Investitionen in die Datenqualität nicht immer zu einer Verbesserung der Kundenbeziehung führen. Doch der untersuchte und modellierte Wirkungszusammenhang erlaubt noch weitergehende Ergebnisse. So können ebenfalls Aussagen über die Entscheidungen und Effekte, die sich für den Anbieter aber auch für den Kunden daraus ergeben, abgeleitet werden. Abschließend sind die Stärken und Schwächen des entwickelten Modells kritisch zu diskutieren sowie zukünftige Forschungsaktivitäten abzuleiten.

\section{2 Überblick über bisherige Ansätze}

Die Verbindung zwischen Datenqualität und Kundenbeziehung wird in einer Reihe von Veröffentlichungen diskutiert [z.B. ArLa02; Dubo01; Eage01; KhHa99; Nels02; Redm96]. Dabei wird häufig (,dogmatisch“) unterstellt, dass eine hohe Datenqualität per se bspw. mittels einer dann möglichen Produktindividualisierung die Kundenzufriedenheit steigert und somit die Beziehung verbessert. Problematisch ist dabei, dass die Beeinflussung durch die Möglichkeiten zur Produktindividualisierung, zur gezielten Kundenansprache oder gar zur höheren Arbeitsmotivation des Verkaufspersonals an Beispielen argumentiert „nachgewiesen“ wird [Redm96]. In welchen Fällen jedoch die Datenqualität überhaupt signifikante Wirkung auf die Entscheidung des Kunden über einen (Folge-)Kauf oder die Fortführung der Beziehung besitzt, wird oftmals nicht oder nur undifferenziert erläutert. Für eine Betrachtung der Effektivität der durchgeführten Datenqualitätsmaßnahmen wäre jedoch genau dies als Basis zwingend nötig. Hierfür mögen zwei Gründe wesentlich sein:

Einerseits fehlt bislang ein einheitliches Qualitätsverständnis bzw. eine allgemein anerkannte Qualitätsdefinition, die als Grundlage für die Forschung und somit für die Herstellung des Zusammenhangs zum CRM hilfreich wäre. So werden die Begriffe Daten- und Informationsqualität häufig synonym verwendet und in der Literatur vielfach beschrieben und untersucht [Helf02; Wan+95]. Das Ergebnis dieser Arbeiten ist eine Vielzahl von Beschreibungs- und Definitionsansätzen sowie Kriterienlisten und Einordnungsrahmen für unterschiedliche Anwendungsgebiete. Aufbauend darauf spezifizieren eine Reihe allgemeiner Ansätze für Datenhaltungs-, Informations- und Wissensmanagementsysteme [Wan+99; Engl99; Wolf99; Redm96], systemspezifische Ansätze für Data-Warehouse-Systeme [Jar+00; Helf02; Hinr02] und internetbasierte [NaRo99] bzw. kooperative Informationssysteme [Cap+03], wie speziell Datenqualität gemessen und gesteuert 
werden kann. Eine grobe Übereinstimmung dieser Ansätze lässt sich dahingehend erkennen, dass Datenqualität anwender- und anwendungsbezogen betrachtet und meist hinsichtlich des Beitrags zur Erreichung der Ziele des Datenverwenders bestimmt wird. Bspw. auf der allgemeinen Qualitätsdefinition nach DIN basierend [DIN95] wird danach der Begriff durch eine Reihe von Qualitätskriterien konkretisiert, die in ihrer Bedeutung und Intensität erheblich vom Anwendungskontext abhängen. Häufig werden die Kriterien Korrektheit, Vollständigkeit, Konsistenz, Relevanz und Aktualität sowie Interpretierbarkeit, Verständlichkeit, Verfügbarkeit und Datensicherheit genannt [z.B. Wan+95].

Daneben bieten die genannten Ansätze für das Datenqualitätsmanagement mit ihren zentralen Komponenten qualitätsverbessernde Prozesse, Organisationsstrukturen und Messansätze zur Datenqualitätsbestimmung abermals eine wichtige Grundlage. Dies ist jedoch einzuschränken, da die Ansätze derzeit oftmals noch unvollständig, generalisiert beschrieben oder lediglich in Teilen konkret ausformuliert sind. Wenngleich somit eine allgemeine Basis existiert, lässt sich dennoch keine Systematik in den Qualitätskriterien, deren Begriffsbestimmung und Dependenzen erkennen, um die für das CRM relevanten Kriterien und Zusammenhänge abzuleiten. Letzteres führt zu der Einschätzung, dass die Datenqualität im Kontext des CRM und den daraus abzuleitenden anwendungsspezifischen Datenqualitätsanforderungen als wenig untersucht bzw. fundiert zu charakterisieren ist. Jedoch wäre dies notwendig, um den zu Beginn in der Literatur behaupteten und unterstellten Wirkungszusammenhang zwischen Datenqualität und Kundenbeziehung erst zu begründen.

Neben der Datenqualität ist andererseits natürlich ebenso zu hinterfragen, was eigentlich unter einer beziehungsorientierten Interaktion verstanden werden kann, gerade im Gegensatz bzw. in Ergänzung zu einer rein transaktionsorientierten Interaktion, welche ausschließlich den Verkauf einzelner Produkte fokussiert. In der Literatur existieren eine Reihe von Definitionen und Vorstellungen über das Konstrukt der Kundenbeziehung. Von zahlreichen Autoren wird dabei auf das notwendige Kriterium verwiesen, dass eine Beziehung als Folge von wechselseitig in Verbindung stehender, „,nicht zufälliger“ Transaktionen zu verstehen ist [Grön99; Plin97; RuOl94]. Deswegen sei sie mehr als „ganzheitliche, fortlaufende“ Interaktion denn als einzelne, eindeutig voneinander abgrenzbare Käufe zu charakterisieren. Was macht jedoch diese „innere Verbindung“ aus bzw. welche Relevanz muss diese besitzen, damit man überhaupt von einer Beziehung sprechen kann?

Hierüber existieren zahlreiche, teilweise auch sehr unterschiedliche Meinungen. Viele Veröffentlichungen betonen, wie bspw. [ErFj01], dass ,,a series of transactions gradually transforms into a relationship, as a result of the social exchange between buyer and seller. A relationship is thus something much more than a series of transactions, and contains dimensions of power, cooperation, commitment, and trust to name but a few". Andere Autoren unterstreichen demgegenüber die 
langfristige ökonomische Zielsetzung der Akteure und eine damit korrespondierende investive Komponente („switching costs“) [Dill96], die ihrerseits bei einem Beziehungsende als „sunk costs“ verloren gehen. Dagegen wird in anderen Beiträgen zwar ebenfalls die Existenz von Ausstiegsbarrieren im Sinne verschiedener Kostengrößen (Such-, Lernkosten, emotionale Kosten) oder Risikofaktoren als Merkmal einer Beziehung angeführt, jedoch ihre Langfristigkeit als notwendiges Kriterium gerade abgelehnt [z.B. Sto+99].

Analog zu dieser kurzen Diskussion lassen sich in der Literatur eine Reihe weiterer Quellen finden, die teilweise gegensätzliche Kriterien und Fälle aufzeigen, in welchen Beziehungen existieren (könnten) bzw. gerade nicht existieren [z.B. Palm96]. Insofern resümieren auch O’Malley und Tynan: „Despite more than ten years of academic and practitioner interest in this area, understanding of the nature of business to consumer relationships has advanced little. [...] Given the diversity in operational approaches employed, and the lack of accepted definitions, it has become impossible to delimit the domain. The boundaries are completely permeable and elastic.” [O’MTy00].

Auch die Diskussion des Begriffs der Kundenbeziehung zeigt, dass eine einheitliche und hier verwendbare Basis nicht direkt übernommen werden kann. Deswegen erscheint es den Autoren sehr wichtig, in den nächsten beiden Abschnitten eine theoriegetriebene, konzeptionelle Grundlage für die Begriffe Datenqualität und Kundenbeziehung zu schaffen.

\section{Spezifikationsorientierte Datenqualitätsdefinition}

Wie eben erläutert, existiert bislang weder eine einheitliche Datenqualitätsdefinition noch eine klare Abgrenzung relevanter Datenqualitätskriterien. Im Folgenden ist daher zunächst eine Konkretisierung des Begriffes im Kontext des CRM vorzunehmen. Nach [Garv84], der verschiedene Ansätze der allgemeinen Qualitätsliteratur systematisiert, bestehen je nach Zielsetzung unterschiedliche Qualitätssichten. So repräsentieren anwenderbezogene Ansätze die Zweckeignung eines Produktes während des Gebrauchs. Produktbezogene Ansätze berücksichtigen inhärente Produkteigenschaften, während herstellungsbezogene Ansätze die Produktionsprozesse in den Mittelpunkt stellen. Demgegenüber beziehen wertbezogene Ansätze Kosten- und Nutzenaspekte ein und betrachten Qualität unter Effizienzgesichtspunkten. Aufbauend auf diesen Sichten können zwei wesentliche Qualitätsfaktoren abgeleitet werden: Designqualität und Konformitätsqualität [Tebo91; Segh96]. Designqualität bezieht sich auf die Übereinstimmung zwischen anwenderbezogenen Anforderungen und deren Konkretisierung in einer Spezifikation. Demgegenüber umfasst Konformitätsqualität die Einhaltung dieser Spezifikation durch Produktionsprozesse. In der Datenqualitätsliteratur wird die Differenzierung in unterschiedliche Qualitätssichten und Qualitätsfaktoren bislang meist nicht vor- 
genommen [vgl. Helf02]. Jedoch würde gerade eine derartige Unterscheidung die zentralen Aufgaben des operativen Datenqualitätsmanagements - die Definition und Erfüllung von Datenqualitätsanforderungen - einbeziehen und sinnvoll abgrenzen [HeHe02]. Es erscheint daher zweckmäßig, die Unterscheidung zur Konkretisierung des Datenqualitätsbegriffs zu berücksichtigen.

Integrierte Informationssysteme, wie sie häufig im CRM Anwendung finden, können grob in die Komponenten operative Anwendungssysteme, Datentransformation und Datentransfer, Datenhaltung sowie Datenanalyse unterschieden werden. Die Festlegung (explizit oder implizit) der Anforderungen an die Komponenten des Informationssystems zum Zeitpunkt $t$ soll durch die Spezifikation $I_{t}^{\text {spez }}$ beschrieben sein (z.B. Datenmodelle mit den zu erfassenden Attributen, die zur Bildung von Kundensegmenten, zur Individualisierung von Leistungen [FrSt01] oder zur unkomplizierten Durchführung von Geschäftstransaktionen benötigt werden [ArLa02; Dubo01]). Demgegenüber wird das vom Informationssystem bereitgestellte Datenangebot zum Zeitpunkt $t$ als $I_{t}^{\text {angebot }}$ bezeichnet (z.B. die konkreten Kundendaten). Es sei angemerkt, dass $I_{t}^{\text {spez }}$ und $I_{t}^{\text {angebot }}$ aufgrund einer ungenügenden Implementierung (z.B. bestimmte Attributwerte sind unvollständig oder inkorrekt erfasst) i.a. nicht deckungsgleich sind. Eine Datennachfrage (i.a. eine Teilmenge des Informationsbedarfs) eines Datenverwenders $u$ zum Zeitpunkt $t$ soll dagegen als $I_{t, u}$ nachfrage bezeichnet sein. Auch hier sei angemerkt, dass $I_{t}^{\text {spez }}$ und $I_{t, u}{ }^{\text {nachfrage }}$ i.a. nicht übereinstimmen (bspw. wird ein Attributwert nachgefragt, der im Datenmodell nicht berücksichtigt ist). Eine (normierte) Qualitätsfunktion des Datenverwenders $u$ zum Zeitpunkt $t$ soll dann die Designqualität als $Q_{t, u}{ }^{\text {design }}\left(I_{t}^{\text {spez }}, I_{t, u}{ }^{\text {nachfrage }}\right) \rightarrow[0 ; 1]$ beschreiben, wobei 0 keine Qualität und 1 eine maximale Qualität bezeichnet. Dagegen beschreibt eine vom Datenverwender nicht abhängige (normierte) Qualitätsfunktion $Q_{t}^{\text {konform }}\left(I_{t}^{\text {spez }}, I_{t}^{\text {angebot }}\right) \rightarrow[0 ; 1]$ die Konformitätsqualität zwischen Spezifikation und Datenangebot, wobei wiederum 0 keine Qualität und 1 eine maximale Qualität bezeichnet. Dabei ist davon auszugehen, dass eine steigende $I_{t}^{\text {spez }}$ zu einer höheren Designqualität $Q_{t}^{\text {design }}$ und eine steigende $I_{t, u}$ nachfrage $_{\text {zu einer geringeren }} Q_{t}^{\text {design }}$ führen (auf Ausnahmen ist noch einzugehen). Analoges gilt für die Konformitätsqualität $Q_{t}^{\text {konform }}$, wobei eine steigende $I_{t}^{\text {spez }}$ zu einer geringeren $Q_{t}^{\text {konform }}$ und ein steigendes $I_{t}^{\text {angebot }}$ zu einer höheren $Q_{t}^{\text {konform }}$ führt.

Datenqualitätsmanagement hat zum Ziel die Gesamtdatenqualität $Q_{t}^{\text {gesamt }}$, die sich aus Designqualität und Konformitätsqualität ergibt, über alle definierten Anwendungsfälle zu optimieren [Helf02]. Dies bedeutet mit anderen Worten, die vom Datenverwender abhängigen Anforderungen in einer Spezifikation zu erfassen, um sie bestmöglichst beim Bau und Betrieb des Informationssystems zu erfüllen. Die allgemeine Zielfunktion des Datenqualitätsmanagements (ohne Gewichtung) zu einem Zeitpunkt $t$ lässt sich dann als

$$
\max Q_{t}^{\text {gesamt }}=\sum_{\mathrm{u}} \mathrm{Q}_{\mathrm{t}, \mathrm{u}}^{\text {design }}\left(\mathrm{I}_{\mathrm{t}}^{\text {spez }}, \mathrm{I}_{\mathrm{t}, \mathrm{u}}^{\text {nachrage }}\right)+\mathrm{Q}_{\mathrm{t}}^{\text {konform }}\left(\mathrm{I}_{\mathrm{t}}^{\text {spez }}, \mathrm{I}_{\mathrm{t}}^{\text {angebot }}\right)
$$


formulieren, wobei Effizienzbetrachtungen hier unberücksichtigt bleiben. Die generellen Optimierungsvariablen bilden dabei $I_{t}^{\text {spez }}, I_{t}^{\text {angebot }}$ und $I_{t, u}{ }^{\text {nachfrage }}$. Da die Datennachfrage $I_{t, u}{ }^{\text {nachfrage }}$ nur durch die Datenverwender in den Geschäftsbereichen beeinflusst werden kann, können Qualitätsverbesserungen direkt nur durch

(a) eine Optimierung der Spezifikation $I_{t}^{\text {spez }}$ oder

(b) einer (qualitativen) Erhöhung des Datenangebots $I_{t}^{\text {angebot }}$

erfolgen. Fall (a) bezieht sich auf Fragen der Informationsbedarfsanalyse und der Spezifizierung von Anforderungen, die insbesondere Auswirkungen auf die Designqualität haben. Diese sollen allerdings nicht Gegenstand dieser Arbeit sein. Demgegenüber umfasst Fall (b) Maßnahmen, die eine Steigerung der Konformitätsqualität bewirken. Grundsätzlich existieren drei Möglichkeiten zur Veränderung des Datenangebots $I_{t}^{\text {angebot }}$, die für das im Weiteren zu entwickelnde Modell elementar sind:

1. Eine Erhöhung des Datenangebots $I_{t}^{\text {angebot }}$ kann durch Maßnahmen zur Erschließung und Nutzung von Datenquellen erfolgen (z.B. Vervollständigung mittels neuer Kundendaten). Diese Maßnahmen sollen im Folgenden durch die Variable $D_{S U P} \in[0 ; 1]$ repräsentiert werden, wobei $D_{S U P}=0$ bedeutet, dass keine der geforderten Daten vorhanden sind. Dagegen seien bei $D_{S U P}=1$ alle benötigten Kundendaten vorhanden. Wird die Erweiterung des Datenangebots aus kundenexternen Quellen („Kauf“ von Daten) nicht berücksichtigt, hängt das Datenangebot insbesondere von den durchgeführten Transaktionen ab, da diese die Basis für die Datenerhebung bilden. Weiter kann davon ausgegangen werden, dass die ersten Transaktionen mit einem Kunden die größte Zunahme an Daten (Adressdaten, „grundlegende“ Bedürfnisse etc.) bewirken. Zusätzliche Transaktionen, insbesondere wenn sie zu früheren identisch sind, werden dagegen einen im Verhältnis dazu geringeren Zuwachs in der Datenmenge zur Folge haben (z.B. nur noch Transaktionsdaten zur Abbildung der Historie der Kundenkontakte). Somit lässt sich für die Datenmenge $D_{S U P}$ in Abhängigkeit der durchgeführten Geschäftstransaktionen eine abnehmende Grenzzunahme begründen.

2. Eine qualitative Erhöhung des $I_{t}^{\text {angebot }}$ kann durch Maßnahmen zur Datenbereinigung im Sinne einer Steigerung der Korrektheit erfolgen. Diese sind dem reaktiven Datenqualitätsmanagement zuzuordnen. Hier ist insbesondere das Data Cleansing anzuführen, das die Standardisierungen und Vereinheitlichungen von heterogenen Daten (z.B. Adressdaten), die Zusammenführung von gleichen Datenobjekten (Record Matching) oder die Überprüfung von semantischen Integritätsbedingungen betrifft [vgl. Jark00].

3. Eine qualitative Erhöhung des $I_{t}^{\text {angebot }}$ ist zudem durch Maßnahmen zur Prozessverbesserung im Sinne einer Steigerung der Vollständigkeit und Korrektheit zu erreichen (z.B. Qualitätsverbesserung durch Änderung der Erfassungs- 
prozesse oder Datentransferprozesse), die dem proaktiven Datenqualitätsmanagement zuordenbar sind [vgl. Helf02].

Die Aspekte 2 und 3 sollen im weiteren durch die Variable $D_{Q M} \in[0 ; 1]$ repräsentiert werden, wobei $D_{Q M}=0$ bedeutet, dass weder Datenbereinigungen noch hochwertige Datenqualitätsprozesse durchgeführt werden bzw. $D_{Q M}=1$ bedeutet, dass das Niveau maximal sei. Bei einer durch die Informationsbedarfsanalyse vorgegebenen $I_{t}^{\text {spez }}$ kann folglich die Konformitätsqualität in Bezug auf die hier fokussierten Kundendaten als

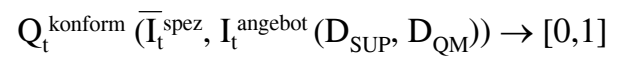

beschrieben werden.

\section{Definition einer Beziehung aus Kundensicht}

Neben dem Begriff der Datenqualität ist es gleichermaßen notwendig den Begriff der Kundenbeziehung, wegen des in der Literatur divergenten und teilweise sehr diffusen Verständnisses, zu bestimmen. Dies soll es erlauben, die Verbindung zur Datenqualität transparenter aufzuzeigen. Als Ausgangspunkt wird die Perspektive des Kunden gewählt [vgl. z.B. Egge99]. Der Grund hierfür ist, dass in gesättigten Märkten nicht das Unternehmen (autonom) bestimmen kann, zu welchen Kunden es gerne eine Geschäftsverbindung aufbauen möchte, sondern der Kunde, insbesondere der aus Anbietersicht „wertvolle“ Kunde, wählt sich seine Geschäftsverbindung(en) aus. Insofern ist es in einem ersten Schritt notwendig zu untersuchen, in welchen Fällen es aus Sicht des Kunden sinnvoll erscheint, von einer beziehungsorientierten statt einer rein transaktionsorientierten Interaktion zu sprechen.

Für das zu entwickelnde Modell, in welches später ebenfalls die Datenqualitätsdefinitionen integriert werden, sollen folgende Prämissen gelten:

1. Der rational-agierende Kunde besitzt eine Nutzenpräferenzfunktion unter Sicherheit, d.h. er soll jeder Angebotsalternative $\mathrm{a}_{\mathrm{i}} \in \mathrm{A}$ (Alternativenraum) eines Anbieters mittels der Abbildung $\Phi: \mathrm{A} \rightarrow \Re$ reelle Nutzenwerte $\Phi(\mathrm{a})$ zuordnen können. Hierdurch kann eine Wertrangfolge verschiedener Produkt- oder Leistungsalternativen definiert werden. Eine Alternative $a_{i}$ zur Durchführung einer Geschäftstransaktion ist demzufolge gegenüber einer anderen Alternative $\mathrm{a}_{\mathrm{k}}$ [superior/inferior/äquivalent], wenn der Nutzenwert $\Phi\left(\mathrm{a}_{\mathrm{i}}\right)$ jeweilig $[>/</=]$ im Vergleich zum Nutzenwert $\Phi\left(a_{k}\right)$ ist.

2. Der (mehrmalig) innerhalb der Betrachtungsperiode auftretende Kundenbedarf sei homogen (z.B. mehrere „gleichartige“ Geldanlage- oder Treibstoffbedarfe). 
Mittels der Präferenzfunktion sind nicht nur die zur Bedarfsbefriedigung angebotenen Kernprodukte, sondern auch zusätzliche Leistungselemente, wie Freundlichkeit des Personals, Anbieterimage etc. zu bewerten, die innerhalb einer Transaktion vom Kunden als nutzenstiftend wahrgenommen werden.

3. Der Kunde besitzt keine Zeitpräferenzen, d.h. bei Leistungsalternativen bzw. Geschäftstransaktionen, deren Nutzen erst in zukünftigen Perioden realisiert wird, kann auf eine zeitadäquate Bewertung (z.B. mittels Barwerten) verzichtet werden.

4. Es wird ein stetiges Modell unterstellt. Bspw. können durch reaktive und proaktive Datenqualitätsmaßnahmen (s.o.) nicht nur bestimmte, diskrete Werte für $D_{S U P}$ und $D_{Q M}$ erzeugt werden. Zudem sei das gesamte Transaktionsvolumen, welches durch die Befriedigung des Kundenbedarfs entsteht, beliebig teilbar. Insofern maximiert der Kunde seinen Nutzen, indem er eine Entscheidung über die Transaktionsanteile je Anbieter an seinem gesamten, nachgefragten Transaktionsvolumen trifft.

Will ein Kunde (in der Betrachtungsperiode) insgesamt $T$ Leistungskäufe bzw. Transaktionen zur Bedarfsbefriedigung durchführen, so sollen diese sein gesamtes Transaktionsvolumen darstellen. Er kann die Transaktionen bei I unterschiedlichen Anbietern durchführen, wobei in der Literatur per Definition häufig die Prämisse des „entweder-oder-Falls“ gesetzt wird, d.h. entweder werden alle oder gar keine Transaktion bei einem Anbieter durchgeführt (vgl. bspw. [Plin97] für den Industriegütermarkt). Zumindest in Endkonsumentenmärkten, wie bspw. im Privatkundengeschäft von Banken, wirkt diese Festlegung zwar vereinfachend, sie ist jedoch keineswegs realistisch (z.B. besitzen bei Sparkassen durchschnittlich ca. 48\% der Kunden und sogar 71\% der einkommensstarken Kunden mehrere Bankverbindungen [Dani01]). Deswegen wird hier der „sowohl-als-auch-Fall“ untersucht, d.h. der Kunde kann seine homogenen Transaktionen auf die Anbieter aufteilen (für nicht-homogene Transaktionen siehe [Hein02]). Für jeden Transaktionsanteil $\lambda_{i}$ bei einem Anbieter $i \in I$ kann der Kunde durch seine Präferenzfunktion zum einen den direkt zurechenbaren Bruttonutzenwert $U_{i}\left(\lambda_{i}\right)$ durch Zuteilung der Transaktionen ermitteln. Andererseits sind für den Kauf, die Inanspruchnahme und Verwertung der angebotenen Leistung die Kosten $K_{i}\left(\lambda_{i}\right)$ für den Transaktionsanteil zu kalkulieren. Insgesamt ergibt sich im Kundenkalkül somit die Aufgabe einer optimalen Wahl des Transaktionsanteils $\lambda_{i}$ für jeden Anbieter $i \in I$ mit $\sum_{\mathrm{i}} \lambda_{i}=1$ (alle $T$ Transaktionen sollen abgewickelt werden), indem die Summe der Nettonutzenwerte $e_{i}$ (Bruttonutzen $U_{i}$ abzüglich der Kosten $K_{i}$ ) maximiert wird.

$$
\begin{aligned}
& \max \mathrm{e}=\sum_{\mathrm{i}} \mathrm{U}_{\mathrm{i}}\left(\lambda_{\mathrm{i}}\right)-\mathrm{K}_{\mathrm{i}}\left(\lambda_{\mathrm{i}}\right) \\
& \text { mit: } \sum_{\mathrm{i}} \lambda_{\mathrm{i}}=1
\end{aligned}
$$

Das Kalkül in Gleichung (2) könnte bspw. als sogenannter „Tankstellenfall“ verstanden werden, bei welchem der Kunde mehrere, völlig isoliert voneinander zu 
beurteilende Kraftstoffkäufe tätigt. Es sind demzufolge nur diejenigen Nutzenund Kostenwerte zu berücksichtigen, die in Entstehung und Wirkung einer Transaktion direkt zugerechnet werden können. Zur Optimierung sind dementsprechend unterschiedliche Transaktionsanteile $\lambda_{i}$ je Anbieter i mit $\lambda_{i} \in[0 ; 1]$ festzulegen. Nur bei konstanten Nutzen- und Kostenwerten je Transaktion, d.h. der Anbieter ändert bspw. den Preis nicht, ergibt sich $\lambda_{i} \in\{0,1\}$, was dem obigen „entwederoder-Fall" entspricht.

Was passiert jedoch nunmehr, wenn Nutzenwerte und Kosten existieren, die in ihrer Entstehung und/oder positiven bzw. negativen Nutzenwirkung nicht mehr direkt einer einzelnen Transaktion, sondern mehreren Transaktionen oder der gesamten Geschäftsverbindung zuzuordnen sind. Sie sollen im weiteren als Verbundeffekte $V$ bezeichnet werden. Verbundeffekte entstehen dabei durch den unmittelbaren oder mittelbaren Kontakt zwischen Kunde und Anbieter. Hierzu gehört bspw. auch, wenn ein anderen Kunden („Mittler“) eine Empfehlung (positive Wirkung) für den Anbieter gibt. Dies bedeutet, dass der Kunde Aktionen durchführt, um sich bewusst oder unbewusst für die Gegenwart oder Zukunft unter Berücksichtigung der (potenziellen) Geschäftsverbindungen zu allen anderen Anbietern einen Nutzen zu erschließen bzw. Kosten zu vermeiden.

Hinsichtlich der Entstehung von Verbundeffekten ist zu unterscheiden:

Verbundeffekte für den Kunden können zum einen ohne Einwirkung und Absicht des Anbieters entstehen, d.h. sie sind in der Entstehung nicht Ausdruck eines geplanten Anbieterhandelns (zum Wiederkaufverhalten ohne Einwirkung des Anbieters vgl. [Riec95]). Hierzu gehören kundenimmanente Aspekte wie (nicht induzierte) Gewohnheit oder Trägheit, die einen Folgekauf basierend auf bereits durchgeführten Transaktionen bei einem Anbieter attraktiver erscheinen lässt. Ein derartiger Nutzenzuwachs wird jedoch nicht konstant über eine größere Transaktionsanzahl sein, da neben dem Gewöhnungseffekt auch ein Abwechslungsbedürfnis des Kunden existiert, d.h. der Kunde zieht aus dem Anbieterwechsel selbst einen Nutzen. Dieses Verhalten wird in der Literatur unter dem Begriff „variety seeking“ diskutiert [McAl82] und wirkt abschwächend und gegenläufig zum Gewöhnungseffekt. Ohne detailliert auf diese Art der Verbundeffekte (im weiteren als Verbundeffekte $V_{H, i}$ beim Anbieter i bezeichnet) im Kontext der Datenqualität einzugehen, lässt sich dennoch festhalten: Unbenommen davon, dass die Verbundeffekte $V_{H, i}$ ohne Einwirkung des Anbieters entstehen, sind sie dennoch im Kundenkalkül zu berücksichtigen. Der Gewöhnungseffekt bedeutet dabei einen positiven Nutzenzuwachs für den Kunden bei steigenden Transaktionsanteilen. Das Abwechslungsbedürfnis schmälert bzw. negiert diesen Nutzenzuwachs bei steigenden, höheren Transaktionsanteilen, was in der Summe einen abnehmenden Grenznutzen (konkaver Kurvenverlauf) für die Verbundeffekte $V_{H, i}(\lambda)$ nach sich zieht. 
Demgegenüber entstehen Verbundeffekte (im Weiteren als Verbundeffekte $V_{A}$ bezeichnet) natürlich insbesondere durch geplantes Anbieterhandeln. Der Anbieter generiert dabei gezielt Nutzenwerte für den Kunden, die nicht auf eine einzelne Transaktion oder Interaktion rückführbar sind, sondern transaktionsübergreifend entstehen respektive auf (eine Reihe von) Folgetransaktionen wirken. Exemplarisch sind hier die Auswirkungen der oben diskutierten Datenqualitätsmaßnahmen zu nennen. Die Qualität bspw. von Kundendaten zu verbessern bedeutet letztlich, sie innerhalb den Transaktionen zielorientiert zu erfassen, danach aufzubereiten und sie letztlich in zukünftigen Transaktionen in adäquater Weise zu verwenden (z.B. zur Individualisierung von Leistungen [ScLi01] oder zur erleichterten, schnelleren Durchführung identischer Transaktionen). Den hierdurch vom Anbieter kreierten Kundennutzen geben die Verbundeffekte $V_{A}$ an.

Die Verbundeffekte $V_{A}$ besitzen wie die erstgenannten Verbundeffekte $V_{\boldsymbol{H}, \boldsymbol{i}}$ eine transaktionsübergreifende Wirkung auf das Kundenkalkül, wie im Fall des für die individuellen Bedürfnisse eines Bankkunden konfigurierten Internetzugangs, der aufgrund der intuitiven Nutzbarkeit den Einarbeitungsaufwand bei Folgetransaktionen senkt. Die Wirkung der Verbundeffekte $V_{A}$ kann dabei in Abhängigkeit des Merkmals konstanter vs. variabler Nutzen mit Bezug auf ein bestimmtes Transaktionsintervall oder übergreifend über alle Transaktionen folgende Ausprägungen besitzen:

Eine Ausprägung von $V_{A}$ sollen die Verbundeffekte $\boldsymbol{V}_{A, C}$ sein, die eine konstante Nutzenwirkung innerhalb eines Intervalls [unterer Grenzwert(LL) $\leq \lambda \leq$ oberer Grenzwert(UL)] des Transaktionsanteils erzielen. Beziehen sich die Verbundeffekte $V_{A, C}$ auf die gesamte Geschäftsverbindung, so entspricht dies einem Intervall von $[0<\lambda \leq 1]$. Werden somit keine Transaktionen durchgeführt $(\lambda=0)$, so realisieren sich ebenso keine (positiven oder negativen) Verbundeffekte $\left(V_{A, C}=0\right)$. Ein Beispiel hierfür sind die Anbahnungskosten für eine Geschäftsverbindung. Demgegenüber können Verbundeffekte $V_{A, C}$ auch erst bei Überschreiten eines Grenzwerts des Transaktionsanteils $\lambda>>0$ existieren. Ein Beispiel hierfür sind die $\mathrm{Zu}-$ sagen des Anbieters über einen fixen, einmaligen Bonus oder Treuerabatt ab einer höheren Transaktionsanzahl.

Demgegenüber sollen die Verbundeffekte $\boldsymbol{V}_{\boldsymbol{A}, \boldsymbol{V}}$ als zweite Ausprägung von $V_{A}$ in ihrer Wirkung nicht konstant sein, sondern sich kontinuierlich mit dem Transaktionsanteil verändern. Ein Beispiel dafür ist die Möglichkeit zur Individualisierung von Leistungen aufgrund sukzessiv gesammelter, qualitativ hochwertiger Kundendaten während fortlaufender Transaktionstätigkeit. Hierauf wird später noch vertieft einzugehen sein, um den Zusammenhang zur Datenqualität transparent darzustellen. Wiederum ist für die Verbundeffekte $V_{A, V}$ eine Intervallabhängigkeit im Sinne von $[\mathrm{LL} \leq \lambda \leq \mathrm{UL}]$ definierbar.

Unter Berücksichtigung der Verbundeffekte ändert sich das obige Kalkül (2) des Kunden wie folgt: 


$$
\begin{aligned}
& \max \mathrm{e}=\sum_{\mathrm{i}} \mathrm{U}_{\mathrm{i}}\left(\lambda_{\mathrm{i}}\right)-\mathrm{K}_{\mathrm{i}}\left(\lambda_{\mathrm{i}}\right)+\mathrm{V}_{\mathrm{H}, \mathrm{i}}\left(\lambda_{\mathrm{i}}\right)+\mathrm{V}_{\mathrm{A}, \mathrm{i}}\left(\lambda_{\mathrm{i}}\right) \\
& \text { mit: } \sum_{\mathrm{i}} \lambda_{\mathrm{i}}=1
\end{aligned}
$$

Die Verbundeffekte $V_{\boldsymbol{H}}$ werden dabei generell aufgrund des Gewöhnungseffekts sowie des Abwechslungsbedürfnisses in der Form

$$
\mathrm{V}_{\mathrm{H}}(\lambda)=\mathrm{V}_{\mathrm{H}} \times(\lambda)^{\mu_{\mathrm{H}}} \text { für } 0<\lambda \leq 1
$$

definiert, wobei $v$ dem Änderungskoeffizient und $\mu$ dem Exponenten der Verbundeffektskurve entspricht (bspw. gilt für den oben diskutierten konkaven Verlauf $0<\mu<1$ ). Demgegenüber lassen sich die Verbundeffekte $V_{A}$ wie folgt generell beschreiben:

$$
\begin{aligned}
\mathrm{V}_{\mathrm{A}}(\lambda)=\mathrm{V}_{\mathrm{A}, \mathrm{V}}+\mathrm{V}_{\mathrm{A}, \mathrm{C}} \\
\text { mit: } \quad \mathrm{V}_{\mathrm{A}, \mathrm{V}}=\mathrm{v}_{\mathrm{A}} \times(\lambda)^{\mu_{\mathrm{A}}} \text { für } \operatorname{LL}(>0) \leq \lambda \leq \mathrm{UL}(\leq 1) \\
\mathrm{V}_{\mathrm{A}, \mathrm{C}}=\text { konst. } \quad \text { für LL }(>0) \leq \lambda \leq \mathrm{UL}(\leq 1)
\end{aligned}
$$

Um die Zusammenhänge besser zu verdeutlichen, wird ein einfaches Beispiel mit zwei Anbietern vorgestellt. Dabei optimiert der Kunde auf Basis gegebener Funktionen ohne dass die Anbieter ihre Output-Größen, wie die Verbundeffekte $V_{A}$ verändern. Dies wird erst später in Verbindung mit den Datenqualitätsmaßnahmen diskutiert.

\section{Anbieter 1:}

$$
\begin{aligned}
& \mathrm{U}_{1}\left(\lambda_{1}\right)=9,45 \lambda \\
& \mathrm{K}_{1}\left(\lambda_{1}\right)=4,6 \lambda \\
& \mathrm{V}_{\mathrm{H}(1)}\left(\lambda_{1}\right)=1,2 \lambda^{0,5}
\end{aligned}
$$

\section{Anbieter 2:}

$$
\begin{aligned}
& \mathrm{U}_{2}\left(\lambda_{2}\right)=8,5 \lambda \\
& \mathrm{K}_{2}\left(\lambda_{2}\right)=3,55 \lambda \\
& \mathrm{V}_{\mathrm{H}(2)}\left(\lambda_{2}\right)=1,2 \lambda^{0,5} \\
& \mathrm{~V}_{\mathrm{A}, \mathrm{C}(2)}\left(\lambda_{2}\right)=0,15 \text { für } 0,8 \leq \lambda_{2} \leq 1
\end{aligned}
$$

Bei den rein transaktionsorientierten Nutzenfunktionen $U_{1}$ und $U_{2}$ wie auch bei den Kostenfunktionen $K_{1}$ und $K_{2}$ ist im Beispiel aufgrund der Prämisse der homogenen Transaktionen ein proportionaler Anstieg definiert (konstante Nutzenwerte und Stückkosten einer Einzeltransaktion). Demgegenüber sei bei den von den Anbietern nicht beeinflussten Verbundeffekten $V_{H(1)}$ und $V_{H(2)}$ ein konkaver, bei beiden Anbietern identischer Verlauf unterstellt. Grund hierfür ist einerseits der durch den Gewöhnungseffekt generierte Zusatznutzen (Prozess ist dem Kunden bekannt und deshalb schneller oder unkomplizierter zu bewerkstelligen). Zum anderen schmälert das Abwechslungsbedürfnis bei höheren Transaktionsanteilen diesen Nutzen ( $\rightarrow$ abnehmender Grenznutzen). In der Ausgangssituation soll darüber hinaus nur noch Anbieter 2 positive Verbundeffekte $V_{A, C(2)}$ in Form einer 
einmaligen Bonuszahlung anbieten. Diese erhält der Kunde jedoch nur für einen Transaktionsanteil $\lambda_{2} \in[0,8 ; 1]$. Von weiteren positiven oder negativen Verbundeffekten, wie z.B. den Anbahnungskosten der Geschäftsverbindung wird zunächst abgesehen.

In einem ersten Schritt wird nur der direkt zurechenbare, isolierte Nettonutzen der Transaktionen (siehe Gleichung (2)) sowie die von den Anbietern nicht beeinflussten Verbundeffekte $V_{H(1)}$ und $V_{H(2)}$ betrachtet. Der von Anbieter 2 offerierte Verbundeffekt (Bonus) bleibt noch unberücksichtigt. Aufgrund des Optimierungskalküls des Kunden lassen sich die Transaktionsanteile $\lambda^{*}{ }_{1}$ und $\lambda^{*}{ }_{2}$ (mit $\lambda *_{1}+\lambda *_{2}=1$ ) folgendermaßen bestimmen:

$$
\begin{aligned}
\max \mathrm{e} & =\mathrm{U}_{1}\left(\lambda_{1}\right)-\mathrm{K}_{1}\left(\lambda_{1}\right)+\mathrm{V}_{\mathrm{H}(1)}\left(\lambda_{1}\right)+\mathrm{U}_{2}\left(1-\lambda_{1}\right)-\mathrm{K}_{2}\left(1-\lambda_{1}\right)+\mathrm{V}_{\mathrm{H}(2)}\left(1-\lambda_{1}\right) \\
& =9,45 \lambda_{1}-4,6 \lambda_{1}+1,2 \lambda_{1}^{0,5}+8,5\left(1-\lambda_{1}\right)-3,55\left(1-\lambda_{1}\right)+1,2\left(1-\lambda_{1}\right)^{0,5} \\
\text { de } / \partial \lambda_{1} & =9,45-4,6+0,6 \lambda_{1}^{-0,5}-8,5+3,55-0,6\left(1-\lambda_{1}\right)^{-0,5} \stackrel{!}{=} 0 \\
& \rightarrow \lambda_{1}^{*} \approx 0,442 \wedge \lambda_{2}^{*} \approx 0,558 \quad\left(\text { mit } \partial^{2} \mathrm{e} / \partial \lambda_{1}<0\right)
\end{aligned}
$$

Als Ergebnis der Optimierung ergibt sich $\lambda^{*}{ }_{1}=0,442$ und $\lambda *_{2}=0,558$. Wird demgegenüber der vom Anbieter 2 offerierte Bonus berücksichtigt und somit die Gleichung (3) zugrundegelegt, so entsteht ein Verbundeffekt für Transaktionsanteile $\lambda_{2} \geq 0,8$. Dies bedeutet, dass zwei intervalldefinierte Nettonutzenfunktionen $e$ für die Bereiche $0 \leq \lambda_{1} \leq 0,2$ (Bonus wird vom Kunden genutzt, da $\lambda_{2} \geq 0,8$ gilt) sowie $0,2<\lambda_{1} \leq 1$ existieren. Optimiert man analog zu oben unter Zugrundelegung des modifizierten Kalküls, so schichtet der Kunde die Anteile der Anbieter auf $\lambda{ }_{1}=0,2$ und $\lambda{ }_{2}=0,8$ um (Wahl der Intervallgrenze - zur Optimalitätsbedingung vgl. [Hein02]). Damit ergibt sich aufgrund der Berücksichtung der Verbundeffekte ein geändertes Bild. Denn wurden zuvor noch 55,8\% der Transaktionen bei Anbieter 2 durchgeführt, so steigt dessen Attraktivität und damit sein Anteil auf $80 \%$. Die Wirkung der Verbundeffekte ( $\lambda^{*}{ }_{1}$ sinkt und $\lambda^{*}{ }_{2}$ steigt!) wird in Abbildung 1 dargestellt.

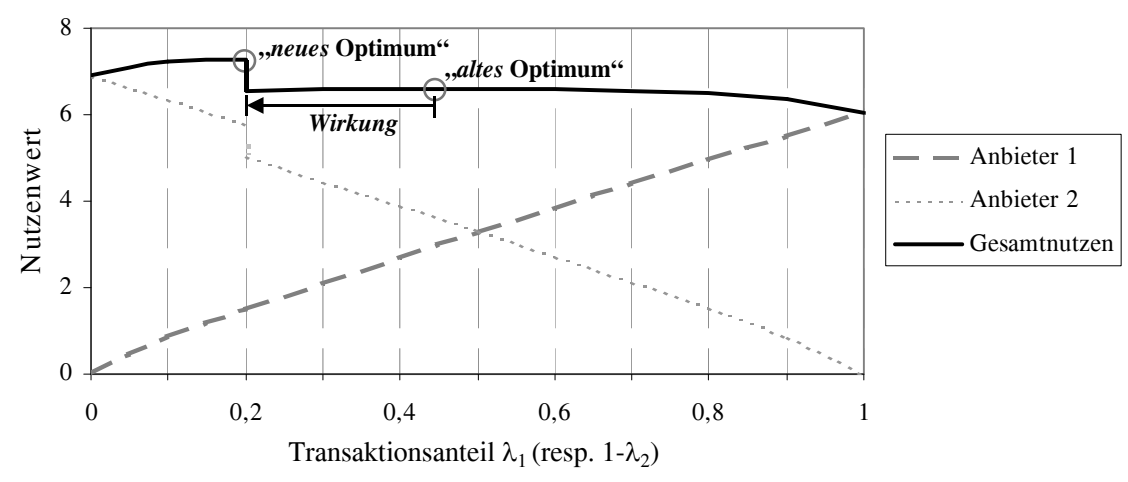

Abbildung 1: Graphische Lösung im 2-Anbieter-Fall 
In der Graphik sind einerseits die jeweiligen Nettonutzenfunktionen der beiden Anbieter eingezeichnet (für Anbieter 2 skizziert im Fall $\lambda_{2}=1-\lambda_{1}$, d.h. an der Achse $\lambda_{1}=0,5$ gespiegelt). Zum anderen ist der sich aus der Kumulation beider Kurven ergebende Gesamtnutzen des Kunden dargestellt. Der Gesamtnutzenverlauf verdeutlicht dabei, dass durch die von Anbieter 2 offerierten Verbundeffekte die Kurve im Intervall $0 \leq \lambda_{1} \leq 0,2$ nach oben „verschoben“ wird. Da die Funktion in diesem Bereich monoton ansteigt und das dadurch resultierende Maximum zugleich einen größeren Nutzenwert besitzt, wie das (lokale) Maximum im Intervall $0,2<\lambda_{1} \leq 1$, entspricht es dem Optimum des Kunden. Wie nunmehr der Anbieter 1 mittels Datenqualitätsmaßnahmen darauf einwirken kann, wird im nächsten Abschnitt zu diskutieren sein.

Zuvor soll jedoch basierend auf den gezeigten Zusammenhängen noch auf den Begriff der Kundenbeziehung eingegangen werden. Es lässt sich feststellen, dass Verbundeffekte nicht mehr darauf abzielen, eine einzelne, isolierte Transaktion vorteilhafter gegenüber einem Konkurrenzangebot darzustellen. Vielmehr „honorieren“ sie in ihrer Wirkung primär eine intensivere (und unter Zugrundelegung mehrerer Perioden eine längere) Geschäftsverbindung, da z.B. eine Anzahl von Folgetransaktionen begünstigt werden. Insofern wird das Konstrukt Kundenbeziehung folgendermaßen definiert:

Eine Beziehung konstituiert sich zwischen Kunde und Anbieter aus Kundensicht aufgrund der Durchführung wenigstens zweier nutzenstiftender Transaktionen, wobei mindestens eine Transaktion durch die Existenz und Relevanz (nicht zwingend Dominanz!) der anbietergenerierten Verbundeffekte $V_{A}$ zustande kommt.

Die Relevanz der anbietergenerierten Verbundeffekte $V_{A}$ (hinreichendes Kriterium für eine Beziehung) begründet sich dabei konkret für den folgenden Fall: Ein unterlegenes Transaktionsangebot nach dem Nettonutzenkalkül der isolierten Transaktion sowie der nicht anbietergenerierten Verbundeffekte wird trotz seiner Suboptimalität vom Kunden gewählt, weil die Nutzendiskrepanz durch die Verbundeffekten $V_{A}$ überkompensiert wird. Verbundeffekte repräsentieren somit die oben angesprochene ,innere“ Verbindung zwischen den Transaktionen (Beziehungscharakter). Im obigen Beispiel trifft dies im Fall des Anbieters 2 für die $\lambda^{*}{ }_{2^{-}}$ Steigerung von 0,558 auf 0,8 zu, da die Verbundeffekten $V_{A}$ hierfür ausschlaggebend sind. Kann hingegen keine zusätzliche Transaktion durch den Einsatz von Verbundeffekten $V_{A}$ seitens eines Anbieters gewonnen werden (im Vergleich zur Situation ohne jegliche Verbundeffekte $V_{A}$ ), so sind die Maßnahmen zur reinen Transaktionsgestaltung (unabhängig von der Höhe existierender Verbundeffekte) für seinen Anteil $\lambda$ ausschlaggebend. Dies bedeutet, die gesamte Interaktion sei als transaktionsorientiert und nicht als beziehungsorientiert charakterisiert. 


\section{Datenqualitätswirkung auf die Kundenbeziehung}

Im Weiteren soll nun die Frage diskutiert werden, ob die Datenqualität Einfluss auf die Kundenbeziehung besitzt und wenn ja, wie sich dieser Zusammenhang modellgestützt darstellen lässt. Die Erkenntnisse des dritten und vierten Kapitels bieten hierfür die Grundlage. Zum einen lässt sich, wie in Kapitel drei gezeigt, die Konformitätsqualität $Q_{t}^{\text {konform }}$ durch drei Maßnahmen verbessern (vgl. Formel (1)): Erstens durch eine Erhöhung des Datenangebots, das in der Variable $D_{\text {SUP }} \in[0 ; 1]$ repräsentiert wird. Zweitens durch Maßnahmen zur Datenbereinigung, die dem reaktiven Datenqualitätsmanagement zuzuordnen sind. Und drittens durch Maßnahmen zur Prozessverbesserung, die dem proaktiven Datenqualitätsmanagement angehören. Die letzten beiden Aspekte werden durch die Variable $D_{Q M} \in[0 ; 1]$ dargestellt. Demgegenüber wurde bei der Diskussion des Konstrukts der Kundenbeziehung die Relevanz der Verbundeffekte als hinreichendes Kriterium definiert (Erhöhung der Beziehungsintensität im Sinne des Transaktionsanteils).

Wie lassen sich nunmehr prinzipiell Verbundeffekte durch Datenqualitätsmaßnahmen erzeugen? Ein Kunde wird per se nicht bereit sein, mehr Daten über sich zur Verfügung zu stellen, ohne den Willen, ein konkretes Bedürfnis durch den Anbieter befriedigen zu lassen. Dies wird er jedoch in der Ausgangssituation nicht tun, da er sich bereits in seinem „Optimum“ befindet. Deswegen muss der Anbieter versuchen, die Konformitätsqualität durch gezielte Qualitätsmaßnahmen zu steigern. Da sich die proaktiven Maßnahmen zur Prozessverbesserung nur auf zukünftig zu erfassende Daten auswirken, müssen Maßnahmen des reaktiven Datenqualitätsmanagements (v.a. Data Cleansing) mit dem Charakter einer Anfangsinvestition ergriffen werden. Bei den Kunden können so, infolge einer „besseren“ Kenntnis über ihre Person, Verbundeffekte $V_{A, V(Q M)}$ kreiert werden, da bspw. eine inhaltlich fundiertere, bedürfnisadäquate Beratung, individuelle Produktangebote oder eine erleichterte, unkomplizierte Durchführung von Geschäftstransaktionen möglich werden. Die durch die Maßnahmen des Datenqualitätsmanagements generierbaren Verbundeffekte seien durch die Funktion

$$
\mathrm{V}_{\mathrm{A}, \mathrm{V}(\mathrm{DQ})}=\mathrm{a} \times \lambda \times \mathrm{D}_{\mathrm{SUP}^{\alpha}}{ }^{\alpha} \mathrm{D}_{\mathrm{QM}^{\gamma}}
$$

dargestellt. Die Parameter $a, \alpha$ und $\gamma$ besitzen dabei in Abhängigkeit vom Kunden(typ) unterschiedliche Ausprägungen und geben generell an, wie ein Kunde(ntyp) die „bessere“ Kenntnis über seine Person bspw. bei einer Beratung schätzt. Grundsätzlich lässt sich jedoch folgendes begründen: Zum einen nimmt der Grenznutzen für den Kunden, wie in Kapitel drei erläutert, bei einer steigenden Datenmenge $D_{S U P}$ kontinuierlich ab ( $\rightarrow \alpha \in(0 ; 1)$ ). Ursache hierfür ist, dass bei Vervollständigung eines existierenden geringen Datenangebots infolge neuer Kundendaten die Nutzenwirkung für den Kunden noch „wahrnehmbarer“ verbessert werden kann („Anbieter weiß offensichtlich mehr über den Kunden“). Im Gegensatz hierzu ist bei Vervollständigung eines schon vorhandenen großen Daten- 
angebots die Grenznutzenwirkung kleiner. Da der Kunde jedoch bisher keine Veranlassung sieht weitere Daten bereitzustellen (er befindet sich im „Optimum“), bleibt $D_{S U P}$ (zunächst) konstant, d.h. Verbundeffekte $V_{A, V(Q M)}$ lassen sich hierüber nicht erzeugen.

Aus diesem Grund ist $D_{Q M}$ durch den Anbieter mittels Maßnahmen des reaktiven Datenqualitätsmanagements initial zu erhöhen. Sie werden, wie auch die Maßnahmen des proaktiven Datenqualitätsmanagements, durch die Variable $D_{Q M}$ ausdrückt. Diese besitzen ebenfalls eine abnehmende Grenznutzenwirkung auf die Verbundeffekte, da Datenqualitätssteigerungen vom Kunden bei einer geringen Datenqualität in größerem Umfang wahrgenommen und honoriert werden, als weitere Steigerungen einer bereits existierenden hohen Datenqualität $(\rightarrow \gamma \in(0 ; 1)$ ). Ein gutes Beispiel wäre das initiale Auffinden und Beseitigen offensichtlich inkorrekter Kundendaten durch Data Cleansing-Maßnahmen, die einen höheren Kundennutzen z.B. durch falsche Kundenansprache bei einer Beratung ermöglichen. Genau wegen dieser Wirkung gilt es auch den Transaktionsanteil $\lambda$ in (6) zu berücksichtigen, da die verbesserte Konformitätsqualität bei jeder (auch potenziell) durchzuführenden Folgetransaktion eine zusätzliche Nutzenwirkung im Sinne der Verbundeffekte erzielt bzw. erzielen könnte. Aufgrund der Prämisse homogener Transaktionen ist der Nutzen pro Transaktion konstant, weswegen der Transaktionsanteil $\lambda$ keinen Parameter als Exponenten erhält.

Bisher hat die Verbesserung der Datenqualität nur eine Wirkung auf die Verbundeffekte $V_{A, V(Q M)}$. Inwiefern hierdurch der Transaktionsanteil erhöht wird, ist mittels der Relevanz der Verbundeffekte zu untersuchen. Dies gibt zugleich eine Antwort auf die Fragestellung „Hat die Datenqualität Einfluss auf die Kundenbeziehung?“. Zur Beurteilung der Relevanz der Verbundeffekte ist zu ermitteln, um welches Niveau die Verbundeffekte $V_{A, V(Q M)}$ mindestens gesteigert werden müssen, um den bisherigen Transaktionsanteil $\lambda^{*}$ des Kunden überhaupt zu erhöhen. Dieses Niveau wird im Weiteren als Signifikanzniveau bezeichnet. Die Datenqualität hat demzufolge keinen Einfluss auf die Geschäftsverbindung (und damit auf den Aufbau einer Beziehung), wenn die ausgelöste Steigerung der Verbundeffekte unter dem Signifikanzniveau liegt. Das Signifikanzniveau lässt sich grundsätzlich durch den Zusammenhang zwischen den Verbundeffekten $V_{A, V(Q M)}$ und dem optimalen Transaktionsanteil $\lambda^{*}$ ermitteln. Hierzu ist für den Fall einer stetigen Funktion das allgemeine Kalkül (3) nach $\lambda$ abzuleiten und zur Ermittlung von $\lambda^{*}$ die Ableitung de/d $\lambda$ Null zu setzen. Da nur $V_{A, V(Q M)}$ als Ansatzpunkt zur Veränderung von $\lambda^{*}$ untersucht wird, bleiben alle anderen Gleichungsgrößen $\left(U(\lambda), K(\lambda), V_{H}(\lambda)\right.$, sonstige $V_{A}(\lambda)$ ) unverändert und sollen hier vereinfachend zum Lageparameter $b$ zusammengefasst werden. Die Gleichung (7) stellt die grundsätzliche Form des Zusammenhangs dar:

1 Liegt das allgemeine Kalkül (3) in der oben definierten Form vor, lässt sich der Zusammenhang zwischen den Verbundeffekten $V_{A, V(Q M)}$ und dem optimalen 


$$
\lambda^{*}=\mathrm{bx} \mathrm{V}_{\mathrm{A}, \mathrm{V}(\mathrm{DQ})^{\beta}}
$$

Eine graphische, idealtypische Verdeutlichung des Zusammenhang zeigt die Abbildung 2. Es wird dabei illustriert, dass bei einem bisherigen Transaktionsanteil $\lambda^{*}{ }_{\text {(alt) }}$ von 0,2 , durch Datenqualitätsmaßnahmen Verbundeffekte $V_{A, V(Q M)}$ mindestens in Höhe von 0,27 erzeugt werden müssen, um den Anteil $\lambda^{*}{ }_{(\text {neu })}$ auf 0,6 zu erhöhen. Gilt hingegen $V_{A, V(Q M)}$ kleiner 0,27, verbleibt der Transaktionsanteil bei $\lambda^{*}{ }_{\text {(alt) }}=0,2$, obwohl für den Kunden ein zusätzlicher Nutzen angeboten wird. Ein Signifikanzniveau entsteht dabei speziell bei intervalldefinierten Nutzenfunktionen (aufgrund bereits vorhandener intervalldefinierter Verbundeffekte $V_{A}$ ) mit zwei oder mehreren (lokalen) Maxima. Hier gibt es die Differenz zwischen dem bisherigen Gesamtnutzenmaximum (an der Stelle $\lambda^{*}{ }_{\text {alt }}$ ) im Vergleich zu einem Nutzenwert an der Stelle $\lambda^{*}{ }_{\text {пеи }}$ an, die der Anbieter erreichen will und kann (vgl. das später folgende Beispiel).

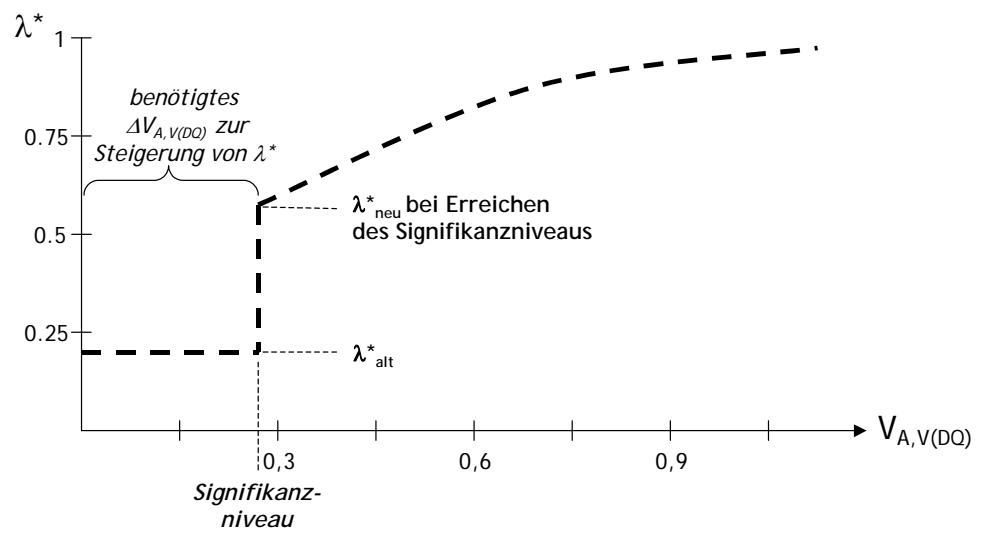

Abbildung 2: Verdeutlichung des Signifikanzniveaus bei Datenqualitätsmaßnahmen

Bei der Diskussion der Datenqualität in Kapitel 3 wurde herausgestellt, dass das Datenangebot $D_{S U P}$ insbesondere vom Transaktionsanteil $\lambda$ abhängig ist (neben der hier nicht betrachteten Möglichkeit externe Datenquellen zu erschließen). Das heißt, steigt der Transaktionsanteil von $\lambda^{*}{ }_{\text {alt }}$ auf $\lambda^{*}{ }_{\text {neu }}$, so können zusätzliche bzw. aktuellere Kundendaten akquiriert werden ( $\rightarrow$ positiven Einfluss auf die Datenqualitätskriterien Vollständigkeit, Aktualität oder Korrektheit). Insofern sei hier die Kundendatenmenge durch die Funktion

$$
\mathrm{D}_{\mathrm{SUP}}=\mathrm{c} \mathrm{x}(\lambda *)^{\sigma}
$$

Transaktionsanteil $\lambda^{*}$ analytisch nicht oder nur intervalldefiniert in die Form von (7) bringen, was jedoch die Aussagen zum Signifikanzniveau nicht beschränkt. 
gegeben. Die Parameter $c$ und $\sigma$ besitzen dabei in Abhängigkeit vom Kunden(z.B. dessen Bereitschaft Daten weiterzugeben) bzw. Transaktionstyp unterschiedliche Ausprägungen. Hierbei lässt sich ein konkaver Verlauf der Funktion begründen, da plausiblerweise zusätzliche Transaktionen eine immer geringere Erhöhung der Datenmenge $D_{S U P}$ besitzen ( $\rightarrow \sigma \in(0 ; 1)$ ). Grund hierfür ist, dass bei der Durchführung weiterer Transaktionen bei einem geringeren im Vergleich zu einem hohen Transaktionsanteil noch mehr neue Kundendaten erhoben werden.

Zugleich bewirkt eine Verbesserung der Kundendatenmenge $D_{S U P}$ aufgrund des erhöhten Transaktionsanteils $\lambda{ }_{\text {nеи }}$ wiederum eine Generierung zusätzlicher Verbundeffekte $V_{A, V(Q M)}$, was die Formel (6) zeigt. Der letztgenannte Zusammenhang bedeutet einen Rückkoppelungs- oder Multiplikatoreffekt, d.h. die ausgelöste Steigerung des Transaktionsanteils hat eine erhöhte Kundendatenmenge zur Folge, welche sich wiederum positiv auf den Transaktionsanteil auswirkt usw.

Zur Verdeutlichung des Einflusses der Datenqualität auf die Geschäftsverbindung bzw. Kundenbeziehung soll das Beispiel aus Kapitel vier fortgeführt werden. Dort hatte der Kunde aufgrund der Nutzen- und Kostenwerte der beiden Anbieter sein Optimum unter Berücksichtigung der bisherigen Verbundeffekte bei $\lambda_{1} *=0,2$ bzw. $\lambda_{2} *=0,8$ ermittelt.

Anbieter 1 entscheidet sich nunmehr für eine (Anfangs-)Investition in die Datenqualität, indem er Data Cleansing-Maßnahmen auf vorhandene jedoch nicht genutzte Kundendaten durchführt. Die Kundendaten wurden deswegen noch nicht genutzt, da Anbieter 1 bislang rein transaktionsorientiert agierte, d.h. die erfassten Daten aus früheren Transaktionen sind nicht als „Value“ der Geschäftsverbindung aufbereitet und in anderen Transaktionen eingesetzt worden. Zu denken ist dabei z. B. an die Transaktions- und Bedürfnishistorie des Kunden, welche zwar in den Datenbanksystemen des Anbieters vorhandenen ist, jedoch noch nicht dem Berater bei einem Kundengespräch zur Verfügung gestellt wird (ein derartiger Sachverhalt ist derzeit z.B. bei einigen Banken durchaus realistisch). Dementsprechend erfolgten bisher keine Maßnahmen des reaktiven und proaktiven Datenqualitätsmanagements (z.B. Datenaufbereitung), was sich im Sachverhalt widerspiegelt, dass $D_{Q M}=0$ ist. Es entstehen in der Ausgangssituation noch keine Verbundeffekte $V_{A, V(Q M)}$.

Für die durchgeführte Investition in Data Cleansing- (reaktiv) sowie Prozessverbesserungsmaßnahmen (proaktiv) wird im Beispiel eine Steigerung von $D_{Q M}$ auf 0,25 angenommenen. Diese soll in Verbindung mit dem Entschluss des Anbieters zur Nutzung der Kundendaten in folgender Funktion Verwendung finden:

$$
\mathrm{V}_{\mathrm{A}, \mathrm{V}(\mathrm{DQ})}=\lambda \times \mathrm{D}_{\mathrm{SUP}}{ }^{0,6} \times \mathrm{D}_{\mathrm{QM}}{ }^{0,5}
$$


Beispielhaft wurden die Parameter $\alpha$ und $\gamma$ auf 0,6 bzw. 0,5 festgesetzt, um den oben diskutierten Zusammenhang eines abnehmenden Grenznutzens der Datenqualität zur Erzeugung von Verbundeffekten zu erhalten. Zudem soll die Gleichung (8), welche die Generierung von Kundendaten in Abhängigkeit vom Transaktionsanteil repräsentiert, die Gestalt

$$
\mathrm{D}_{\mathrm{SUP}}=(\lambda *)^{0,5}
$$

besitzen, d.h. dem Parameter $\sigma$ wird der Wert 0,5 zugewiesen (abnehmende „Grenzgewinnung“ von Kundendaten bei steigendem Anteil homogener Transaktionen). Der Parameter $c$ in Gleichung (8) erhält aufgrund des Zusammenhangs $\lambda^{*}=1 \Rightarrow D_{\text {SUP }}=1$ den Wert 1 , da im Beispiel Kundendaten nur aus Transaktionstätigkeit und nicht aus externen Datenquellen gewonnen werden können. Mit dem bisherigen (vor der Investitionsentscheidung des Anbieters) optimalen Transaktionsanteil von $\lambda_{1} *=0,2$ aus Kundensicht ergibt sich somit ein $D_{S U P}$ von $0,2^{0,5}=0,447$. Mit der Steigerung von $D_{Q M}$ auf 0,25 resultiert $V_{V(Q M)}=0,309 \lambda$. In Analogie zur Kundenoptimierung in (3) ergibt sich somit im ersten Schritt ein verändertes Kalkül (im Intervall $\left.\left.\lambda_{1} \in\right] 0,2 ; 1\right]$ ) mit

$$
\mathrm{e}=9,45 \lambda_{1}-4,6 \lambda_{1}+1,2 \lambda_{1}{ }^{0,5}+\underline{0,309 \lambda_{1}}+8,5\left(1-\lambda_{1}\right)-3,55\left(1-\lambda_{1}\right)+1,2\left(1-\lambda_{1}\right)^{0,5},
$$

welches $V_{A, V(Q M)}$ nunmehr berücksichtigt. Mit diesem modifizierten Kalkül bestimmt der Kunde sein neues Optimum bei $\lambda_{1} *=0,619$ sowie $\lambda_{2} *=0,381$, d.h. der Transaktionsanteil von Anbieter 1 steigt aufgrund des Datenqualitätseffekts um $\Delta \lambda_{1} *=0,419$. Letztgenannter Effekt entsteht aus den Kundendaten aller Transaktionen des bisherigen Anteils von 0,2, er wirkt jedoch wegen der (potenziellen) Nutzbarkeit auf Transaktionen, die den bisherigen Anteil übersteigen. Darum ist beim Datenqualitätseffekt von einem Verbundeffekt zu sprechen. Das oben diskutierte Signifikanzniveau spielt im Beispiel ebenso eine Rolle. Würde Anbieter 1 infolge der Anfangsinvestitionen für $D_{Q M}$ statt 0,25 weniger als 0,18 erreichen, so könnte das hier gültige Signifikanzniveau für $V_{A, V(Q M)}$ in Höhe von 0,276 $\lambda$ (abgeleitet aus (7) nicht überschritten werden (vgl. hierzu Abbildung 2). D.h. es erfolgt keine Veränderung des Transaktionsanteils zugunsten von Anbieter 1. Der Gesamtnutzen für den Kunden an der bisherigen Stelle $\lambda_{1} *=0,2 \mathrm{bzw}$. $\lambda_{2} *=0,8$ wäre immer noch maximal über den gesamten Bereich. Der Datenqualitätseffekt hätte keine Wirkung.

Der neue Transaktionsanteil $\lambda_{1} *$ führt jedoch im nächsten Schritt ohne weiteren Eingriff des Anbieters 1 zu einer Erhöhung der Kundendatenmenge $D_{S U P}$ von bisher 0,447 auf $0,619^{0,5}=0,787$. Dies berücksichtigt der Kunde seinerseits wiederum bei den Verbundeffekten $V_{A, V(Q M)}$, da die nun erstmalig erhöhte Datenmenge (bisher wurde mit der Anfangsinvestition in Data Cleansing-Maßnahmen nur der Parameter $D_{Q M}$ gesteigert) ebenfalls positiven Einfluss auf die Transaktionsdurchführung besitzt. Berechnet man die gestiegene Kundendatenmenge $D_{S U P}$ von 0,787 ein, so ergibt sich für $V_{A, V(Q M)}$ ein geänderter Wert von $0,433 \lambda$, was auf den opti- 
malen Transaktionsanteil $\lambda_{1} *$ rückkoppelt. Diese Rückkoppelung wurde oben bereits als Multiplikatoreffekt der Datenqualität eingeführt. Der gesamte Wirkungsverlauf der Datenqualitätsinvestition (im Sinne einer komparativen Statik) kann folgender Tabelle entnommen werden: 


\begin{tabular}{|c|c|c|c|c|c|}
\hline Schritt & $\mathrm{D}_{\mathrm{QM}}$ & $\mathrm{D}_{\mathrm{SUP}}$ & $\mathrm{V}_{\mathrm{V}(\mathrm{QM})}$ & $\lambda_{1}{ }^{*}$ & $\lambda_{2}{ }^{*}$ \\
\hline 0 & 0 & 0,447 & - & 0,2 & 0,8 \\
\hline 1 & $\begin{array}{c}0,25 \\
\text { (Investition) }\end{array}$ & 0,447 & $0,309 \lambda$ & 0,619 & 0,381 \\
\hline 2 & 0,25 & 0,787 & $0,433 \lambda$ & 0,68 & 0,32 \\
\hline 3 & 0,25 & 0,825 & $0,445 \lambda$ & 0,686 & 0,314 \\
\hline 4 & 0,25 & 0,828 & $0,447 \lambda$ & 0,686 & 0,314 \\
\hline
\end{tabular}

Tabelle 1: Wirkungsverlauf der Datenqualitätsinvestition im Beispiel

Wie die Tabelle 1 zeigt, konvergiert bei einer einmaligen Anfangsinvestition des Anbieters der Transaktionsanteil $\lambda_{1} *$ auf den Wert 0,686, wobei im Vergleich zum Ausgangswert von 0,2 die Differenz von 0,419 auf der initialen Erhöhung von $D_{Q M}$ und die Differenz von 0,067 auf der anschließende Erhöhung von $D_{S U P}$ basiert.

Wie das Beispiel nochmals verdeutlicht, lässt sich ein wechselseitiger Wirkungszusammenhang zwischen einer erhöhten Datenqualität und einer verbesserten Beziehungssituation begründen. Die gesamten Ergebnisse der Arbeit, die Relevanz für die Praxis wie auch den Ausblick fasst der nächste Abschnitt kurz zusammen.

\section{Zusammenfassung der Ergebnisse und Ausblick}

Ziel des Artikels war es, den Einfluss der Datenqualität auf die Kundenbeziehung grundlagenorientiert herauszuarbeiten, um auf die Frage, ob und in welchen Fällen eine Beziehung durch Verbesserung der Datenqualität aufgebaut werden kann, Antworten zu liefern. Hier sollte vor dem Hintergrund einer oftmals undifferenzierten Diskussion der Thematik („Datenqualität ist für CRM per se wichtig und unentbehrlich!“) ein Beitrag zur Klärung der Wirkungszusammenhänge geleistet werden. Die wesentlichen Aussagen sind:

Basierend auf der Begriffsbestimmung von Datenqualität und Kundenbeziehung, lässt sich die Wirkung der Datenqualitätsmaßnahmen im CRM (im Gegensatz zur rein transaktionsorientierten Interaktion) transparent aufzeigen. In der Literatur wird dieser Wirkungszusammenhang bislang meist nur oberflächlich diskutiert und generell ein nachhaltiger, positiver Zusammenhang auf die Verbesserung der Beziehung unterstellt [z.B. KhHa99; Dubo01]. Die Ergebnisse betonen jedoch, dass die Maßnahmen nicht immer zu einer Verbesserung der Geschäftsverbindung aus Anbietersicht führen müssen. Erstens bedarf es hierzu einer Affinität seitens des Kunden, d.h. dieser zieht einen Nutzen aus einer besseren Beratungsmöglichkeit oder einer unkomplizierteren Transaktionsabwicklung etc. aufgrund der Spei- 
cherung und Aufbereitung seiner Daten unter Qualitätsgesichtspunkten. Zum anderen muss der generierte Nutzen ein Signifikanzniveau erreichen, damit die Wirkung der Datenqualitätsmaßnahmen nicht „verpufft“, sondern zu einer intensiveren Beziehung führen kann. Beide Aspekte stammen unmittelbar aus dem Kontext des CRM und dienen als wichtige „Steuerungsgrößen“ für das Datenqualitätsmanagement. Hieraus sind ebenfalls praktische Handlungsempfehlungen abzuleiten, d.h. in welchem Umfang Investitionen ökonomisch sinnvoll sind. Einerseits muss zwar das Signifikanzniveau überschritten werden, zum anderen ist jedoch das Streben nach einer extrem hohen Datenqualität aufgrund der abnehmenden Grenzwirkung (z.B. zwischen den ergriffenen Datenqualitätsmaßnahmen und den damit erzeugten Verbundeffekten) wirtschaftlich ebenso zweifelhaft.

Daneben gilt es im Ergebnis den Multiplikatoreffekt der Datenqualität infolge eines erhöhten Transaktionsanteils und einer vergrößerten Kundendatenmenge festzuhalten („,̈berwindung“ des oder der Signifikanzniveaus). Im Gegensatz zu anderen Verbundeffekt-erzeugenden Beziehungswerten, wie Vertrauen oder monetäre Anreize [vgl. Hein02], tritt bei qualitativ hochwertigen Kundendaten ein Multiplikator auf. Diesen gilt es insbesondere hinsichtlich möglicher praktischer Gestaltungsoptionen noch genauer zu untersuchen. Der Fokus ist hierbei bspw., wie insbesondere durch proaktives Datenqualitätsmanagement eine derartige Rückkoppelung gefördert und intensiviert wird, da sich die (zusätzlich gewonnene) Kundendatenmenge dadurch „qualitätsgerechter“ erfassen lässt [Helf02].

Beim entwickelten Modell sind jedoch eine Reihe von Punkten kritisch zu sehen, aus welchen sich der weitere Forschungsbedarf ableitet:

1. Ein Kritikpunkt ist in der Begrenzung durch die Modellprämissen zu sehen. Zum einen war diese nach Meinung der Autoren jedoch zur Schaffung einer geeigneten theoretischen Grundlage notwendig. Zum anderen ist natürlich zukünftig der Modellkontext zu erweitern. Primär fallen darunter die Berücksichtigung nicht homogener Transaktionen (z.B. unterschiedliche Bankprodukte) sowie die zeitliche Dynamisierung des Modells, d.h. die Anbieter treffen wechselseitig abhängige Entscheidungen zu verschiedenen aufeinanderfolgenden Zeitpunkten.

2. Ein zweite wesentliche Aufgabe ist die Konkretisierung und empirische Messung der oben begründeten funktionalen Zusammenhänge zwischen Datenqualität und Kundenbeziehung (z.B. für verschiedene Kundentypen) sowie der im Modell verwendeten Variablen (vor allem das Datenangebots $D_{S U P}$, die Datenqualitätsmaßnahmen $D_{Q M}$ und die durch Datenqualität generierten Verbundeffekte $\left.V_{A, V(Q M)}\right)$. Hier liegt das Augenmerk besonders auf den Verbundeffekten infolge von Datenqualitätsmaßnahmen wie auch der Schätzung des Signifikanzniveaus und des Multiplikatoreffekts, um effektive Stellgrößen zu bestimmen. Geht man nämlich realistischerweise davon aus, dass unterschiedliche Kunden(typen) nicht über einheitliche Signifikanzniveaus verfügen, so 
stellt sich die Frage, mit welcher Intensität Datenqualitätsmaßnahmen zu ergreifen sind? Da der Einsatz vieler reaktiver und proaktiver Datenqualitätsmaßnahmen in der Praxis oftmals nicht für jeden einzelnen Kunden(typ) selektiv entschieden werden kann, ist eine Abwägung hinsichtlich der richtigen Intensität notwendig. Einerseits deshalb, um bei vielen Kunden nicht unter dem Signifikanzniveau zu liegen und so eine größere Wirkung zu verfehlen. Zum anderen können bei einem intensiven Maßnahmeneinsatz die damit korrespondierende Ressourcennutzung durch den zusätzlich gewonnenen Anbieternutzen nicht gedeckt werden. Somit ergeben sich eine Reihe interessanter, praxisrelevanter Überlegungen für die Steuerung der Datenqualität, die durch empirische Verfahren (z.B. conjoint-Analyse für die Abschätzung des Nutzens der Datenqualität im Sinne der Verbundeffekte) zu fundieren sind.

3. Neben der Effektivität ist ebenfalls die hier bewusst noch nicht erörterte Effizienz der ergriffenen Datenqualitätsmaßnahmen zu betrachten. Dies bedeutet, dass ein Vergleich hinsichtlich des Kosten-/Nutzenverhältnisses zwischen qualitativ hochwertigen Kundendaten als nicht-monetären Beziehungswert und anderen Beziehungswerten (wie z.B. Bonuszusagen des Anbieters) zu ziehen ist. Hier sind die für den Anbieter jeweils zusätzlich realisierbaren Erlöse einer Beziehungsverbesserung (v.a. Transaktionserlöse) den Aufwendungen der ergriffenen Maßnahmen gegenüberzustellen, um wiederum nicht das technische Machbare sondern das ökonomisch Sinnvolle in den Mittelpunkt der Betrachtung zu rücken.

\section{Literatur}

[ArLa02] Arndt, D., Langbein, N. : Data Quality in the Context of Customer Segmentation, in Fischer, C., Davidson B. (Hrsg.): Proceedings of the 7th International Conference on Information Quality, Cambridge 2002, S. 47-60.

[Cap+03] Cappiello, C., Francalanci, Ch., Pernici, B., Plebani, P., Scannapieco, M.: Data Quality Assurance in Cooperative Information Systems: A muliti-dimensional Quality Certificate, in Catarci, T. (Hrsg.): International Workshop on Data Quality in Cooperative Information Systems, Siena 2003, S. 64-70.

[Dani01] Daniel, J.: Ertragssteigerung durch Kundenzufriedenheit und Kundenbindung. Möglichkeit und Grenzen am Beispiel eines Kreditinstituts, Stuttgart 2001.

[Dill96] Diller, H.: Kundenbindung als Marketingziel, in: Marketing ZFP, 1996, Nr. 2, S. 81-94.

[DIN95] Deutsches Institut für Normung e. V.: Qualitätsmanagement, Statistik, Zertifizierung: Begriffe aus DIN-Normen, 2. Aufl., Berlin u.a. 1995.

[Dubo01] Dobois, L.: New Channels Present New CRM Challenge, in The American Banker, 7. Feb. 2001. 
[Eage01] Eager, A.: CRM data needs to come clean, in Computer Business Review, Vol. 9 (2001), Nr. 11, S. 40.

[Ecke02] Eckerson, W.: Data Quality and the Bottom Line, The Data Warehousing Institute Report Series No. 101, Chatsworth 2002.

[Egge99] Eggert, A.: Kundenbindung aus Kundensicht: Konzeptualisierung - Operationalisierung - Verhaltenswirksamkeit, Wiesbaden 1999.

[Engl99] English, L.: Improving Data Warehouse and Business Information Quality, New York u.a. 1999.

[ErFj01] Eriksson, K., Fjeldstad, O.: Value Creation in Financial Services, "The future of retail banking and customer relationships“ - The first workshop of The Göran Collert Research Foundation, Sep. 13-14, 2001.

[FrSt01] Fridgen, M, Steck, W.: New Perspectives on Individualization and Controlling of Web Sites for the Financial Services Industry, Diskussionspapier WI-99, Institut für Betriebswirtschaftslehre, Universität Augsburg, 2001.

[Garv84] Garvin, D. A.: What does "Product Quality” really mean?, in Sloan Management Review, Vol. 26 (1984), Nr. 1, S. 25-43.

[Grön99] Grönroos, C.: The relationship marketing process: interaction, communication, dialogue, value, 2nd conference on relationship marketing, Monash University, 1999.

[Hein02] Heinrich, B.: Methode zur wertorientierten Analyse und Gestaltung der Kundeninteraktion - Zur Rolle des Service Integrators im Privatkundengeschäft von Kreditinstituten, Dissertation, Berlin 2002.

[Helf02] Helfert, M: Proaktives Datenqualitätsmanagement in Data-Warehouse-Systemen. Qualitätsplanung und Qualitätslenkung, Dissertation, Berlin 2002.

[HeHe02] Helfert, M., Herrmann, C.: Proactive data quality management for data warehouse systems, in: 4th Intern. Workshop on 'Design and Management of Data Warehouses' (DMDW'2002) in conjunction with CAiSE 2002, Toronto, 27. Mai 2002, S. 97-106.

[Hinr02] Hinrichs, H.: Datenqualitätsmanagement in Data Warehouse-Systemen, Dissertation, Oldenburg 2002.

[Hua+99] Huang, K., Lee, Y., Wang, R. Y.: Quality Information and Knowledge, Upper Saddle River 1999.

[Jar+00] Jarke, M., Lenzerini, M., Vassiliou, Y., Vassiliadis, P.: Fundamentals of data warehouses, Berlin u.a. 2000.

[KhHa99] Khalil, O. E. M., Harcar, T. D.: Relationship Marketing and Data Quality Management, in SAM Advanced Management Journal, Vol. 64 (1999), No. 2, S. 26-33.

[McAl82] McAlister, L.: A Dynamic Attribute Satiation Model of Variety Seeking Behavior, Journal of Consumer Research, Vol. 9 (1982), S. 141-150. 
[Naum02] Naumann, F.: Quality-Driven Query Answering for integrated Information Systems, Berlin u.a. 2002.

[Nels02] Nelson, S.: What’s happening to CRM in 2002, Gartner Group, January 2002.

[O’MTy00] O‘Malley, L., Tynan, C.: Relationship marketing in consumer markets Rhetoric or reality?, European Journal of Marketing Vol. 34 (2000), Nr. 7, S. 797-815.

[Plin97] Plinke, W.: Grundlagen des Geschäftsbeziehungsmanagements, in: Kleinaltenkamp, M.; Plinke, W.: Geschäftsbeziehungsmanagement, Berlin u.a. 1997, S. 1-61.

[Redm96] Redman, Th. C.: Data Quality for the Information Age, Norwood 1996.

[Riec95] Riecker, S. A.: Bedeutende Kunden, Wiesbaden, 1995.

[RuOl94] Rust, R. T., Oliver, R. W.: The Death of Advertising, Journal of Advertising, Dec. 1994, S. 71-78.

[ScLi01] Schackmann, J., Link, H.: Mass Customization of Digital Products in Electronic Commerce, in Sebaaly, M. F.; Proceedings of the International NAISO Congress on ISI 2001, S.144-150.

[Segh96] Seghezzi, H. D.: Integriertes Qualitätsmanagement: das St. Galler Konzept, München und Wien 1996.

[Sto+99] Storbacka, K., Strandvik, T., Grönroos, C.: Gewinn durch Relationship Management, in Payne, A., Rapp, R. (Hrsg.): Handbuch Relationship Marketing: Konzeption und erfolgreiche Umsetzung, München 1999, S. 69-89.

[Tebo91] Teboul, J.: Managing Quality Dynamics, New York u.a. 1991.

[Wan+95] Wang R.Y., Storey, V.C., Firth, Ch.P.: A Framework for analysis of data quality research, in IEEE Transaction on Knowledge and Data Engineering, Vol. 7 ( 1995), Nr. 4, S. 623-640.

[Wolf99] Wolf, P.: Konzept eines TQM-basierten Regelkreismodells für ein „Information Quality Management“ (IQM), Dortmund 1999. 